\title{
Field Dependence of the Muon Spin Relaxation Rate in MnSi
}

\author{
I. M. Gat-Malureanu, ${ }^{1}$ A. Fukaya, ${ }^{1}$ M. I. Larkin, ${ }^{1}$ A. J. Millis, ${ }^{1}$ P. L. Russo, ${ }^{1}$ A. T. Savici, ${ }^{1}$ Y. J. Uemura, ${ }^{1}$ P. P. Kyriakou, ${ }^{2}$ \\ G. M. Luke, ${ }^{2}$ C. R. Wiebe, ${ }^{2}$ Y.V. Sushko, ${ }^{3}$ R. H. Heffner, ${ }^{4}$ D. E. MacLaughlin, ${ }^{5}$ D. Andreica, ${ }^{6}$ and G. M. Kalvius ${ }^{7}$ \\ ${ }^{1}$ Department of Physics, Columbia University, New York, New York 10027 \\ ${ }^{2}$ Department of Physics and Astronomy, McMaster University, Hamilton, Ontario, Canada L8S 4MI \\ ${ }^{3}$ Department of Physics and Astronomy, University of Kentucky, Lexington, Kentucky 40506 \\ ${ }^{4}$ MS K764, Los Alamos National Laboratory, Los Alamos, New Mexico 87545 \\ ${ }^{5}$ Department of Physics, University of California, Riverside, California 92521 \\ ${ }^{6}$ Institute for Particle Physics of ETH Zurich, CH-5232 Villigen PSI, Switzerland \\ ${ }^{7}$ Physics Department, Technical University of Munich, 85747 Garching, Germany
}

(Received 24 July 2002; published 15 April 2003)

\begin{abstract}
Muon spin rotation/relaxation measurements have been performed in the itinerant helical magnet $\mathrm{MnSi}$ at ambient pressure and at $8.3 \mathrm{kbar}$. We have found the following: (a) the spin-lattice relaxation rate $1 / T_{1}$ shows divergence as $T_{1} T \propto\left(T-T_{\mathrm{c}}\right)^{\beta}$ with the power $\beta$ larger than 1 near $T_{\mathrm{c}}$; (b) $1 / T_{1}$ is strongly reduced in an applied external field $B_{\mathrm{L}}$ and the divergent behavior near $T_{\mathrm{c}}$ is completely suppressed at $B_{\mathrm{L}} \geq 4000 \mathrm{G}$. We discuss that (a) is consistent with the self-consistent renormalization theory and reflects a departure from "mean-field" behavior, while (b) indicates selective suppression of spin fluctuations of the $q=0$ component by $B_{\mathrm{L}}$.
\end{abstract}

DOI: 10.1103/PhysRevLett.90.157201

Manganese monosilicide ( $\mathrm{MnSi}$ ), a magnetic system with itinerant electrons, orders magnetically at a temperature $T_{\mathrm{c}}=29.5 \mathrm{~K}$ into a helical magnetic structure with a long period of $180 \pm 3 \AA$ and a small staggered magnetic moment $M_{0}=0.4 \mu_{\mathrm{B}}$ per Mn at $T=0 \mathrm{~K}$ [1]. In a magnetic field, the magnetic structure transforms progressively from helical to conical to ferromagnetic, as shown in Fig. 1(a). Above $6.2 \mathrm{kG}$ (at $4.2 \mathrm{~K}$ ) the system behaves like a ferromagnet having a relatively large high field susceptibility [3]. In the paramagnetic state the magnetic susceptibility $\chi$ obeys a Curie-Weiss law up to $400 \mathrm{~K}$ [4].

This system has been extensively studied as a prototype of itinerant weak ferromagnets which follow predictions of the self-consistent renormalization (SCR) theory developed by Moriya, Kawabata, and co-workers [5]. The SCR theory overcomes shortcomings of earlier mean field (Stoner-Slater) theory and random phase approximation, and successfully explains the Curie-Weiss behavior of $\chi$, the ordering temperature, and other properties of the metallic ferro- and antiferromagnets, such as $\mathrm{Fe}, \mathrm{Ni}$, and $\mathrm{ZrZn}_{2}$. In a crossover from the magnetism of localized moments to that of itinerant electrons, MnSi is located in a "very itinerant" region where the magnetic moment size $M_{0}$ and $T_{\mathrm{c}}$ are very small, while strong spin fluctuations extend to a rather high energy scale. Recently, Pfleiderer et al. [6] found that, in applied pressure above $p_{\mathrm{c}}=14.6 \mathrm{kbar}, \mathrm{MnSi}$ becomes a correlated paramagnet.

Previously, Hayano et al. [7] performed muon spin relaxation $(\mu \mathrm{SR})$ measurements in $\mathrm{MnSi}$ and reported divergence of the spin-lattice relaxation rate $1 / T_{1}$ observed above $T_{\mathrm{c}}$ in an applied longitudinal field $B_{\mathrm{L}}$ of
PACS numbers: 75.25.+z, 71.27.+a, 75.40.-s, 76.75.+i
$700 \mathrm{G}$, following $T_{1} T \propto\left(T-T_{\mathrm{c}}\right)$. This behavior is consistent with the prediction of the SCR theory in a wide temperature region above $T_{\mathrm{c}}$. Kadono et al. [8] confirmed this result in subsequent $\mu \mathrm{SR}$ studies, which elucidated muon-nuclear double relaxation effects.

In this Letter, we report our new $\mu$ SR measurements on a single crystal of $\mathrm{MnSi}$ under longitudinal magnetic fields $B_{\mathrm{L}} \leq 6 \mathrm{kG}$ in ambient and applied pressure. We discovered the following: (a) a strong dependence of $1 / T_{1}$ on $B_{\mathrm{L}}$; (b) a departure of $T_{1} T$ from the linear behavior in $\left(T-T_{\mathrm{c}}\right)$ at temperatures very close to $T_{\mathrm{c}}$. We will discuss these results in terms of (a) different

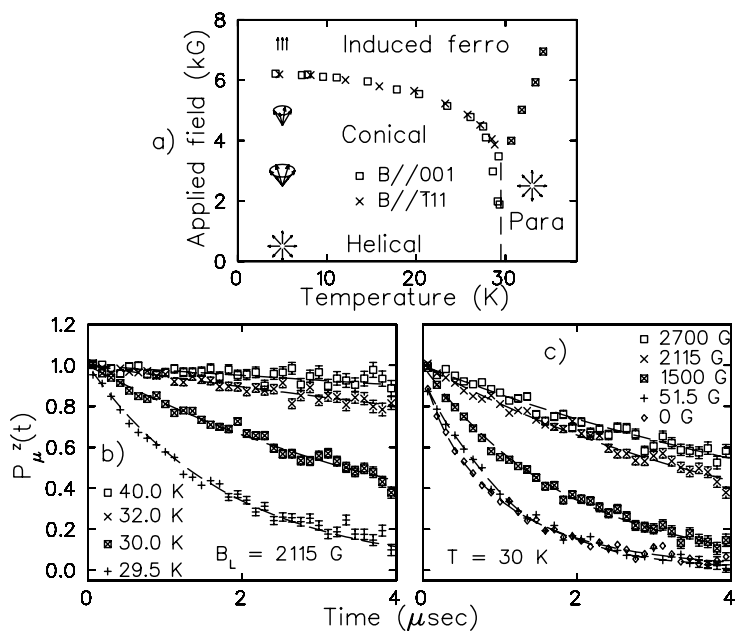

FIG. 1. (a) Field-temperature phase diagram of MnSi [2]. (b) The muon spin polarization function $P_{\mu}^{\mathrm{z}}(t)$ observed in $\mathrm{MnSi}$ at ambient pressure with $B_{\mathrm{L}}=2115 \mathrm{G}$. (c) The field dependence of $P_{\mu}^{\mathrm{z}}(t)$ at $T=30 \mathrm{~K}$. 
effects of $B_{\mathrm{L}}$ on uniform and helical spin fluctuations, and (b) departure from "mean-field" behavior of $\chi$ near $T_{\mathrm{c}}$.

We performed zero field (ZF) and longitudinal field (LF) $\mu$ SR measurements at the M20 and M9 Channels of TRIUMF, Vancouver, Canada. A positive muon beam, polarized along the flight direction, was stopped in a specimen, and histograms of the muon decay positrons, as a function of the residence time $t$ of $\mu^{+}$in the crystal, were recorded by using forward/backward counters. In ambient pressure, we used a beam of momentum of $\sim 28 \mathrm{MeV} / c$ and a single crystal specimen of $8 \times 8 \times$ $2 \mathrm{~mm}^{3}$, with the largest surface perpendicular to the cubic crystal axis placed along the beam direction. For measurements at $8.3 \mathrm{kbar}$, we mounted a larger single crystal in a piston-cylinder pressure cell, in which Daphne oil is compressed to produce uniform hydrostatic pressure, and used a beam of momentum of $\sim 110 \mathrm{MeV} / c$ directed perpendicular to the cubic crystal axis.

Since positrons are emitted preferentially along the muon spin direction, the muon spin polarization function $P_{\mu}^{\mathrm{z}}$ can be obtained from the forward/backward asymmetry in positron histograms. In the paramagnetic state of ferro- or antiferro-magnets $P_{\mu}^{\mathrm{z}}$ is usually given as a product of the Kubo-Toyabe function, $G_{\mathrm{KT}}(t)$, which describes the effect of nuclear dipolar fields, and an exponential function, $\exp \left(-t / T_{1}\right)$, due to electron spin fluctuations. Application of LF with $B_{\mathrm{L}} \geq 50 \mathrm{G}$ would "decouple" nuclear dipolar fields, making $G_{\mathrm{KT}}(t) \approx 1$. In the ordered state $P_{\mu}^{\mathrm{z}}$ consists of an oscillating signal representing muon spin precession around the static internal field, added to the exponentially decaying nonoscillating signal. These two signals appear with the amplitude ratio of 2:1 in ZF- $\mu$ SR using powder samples, while the ratio changes in single crystal samples and/or in LF- $\mu$ SR [9].

In $\mathrm{MnSi}$, previous $\mathrm{ZF}-\mu \mathrm{SR}$ studies [8] found two different precession frequencies, indicating two different muon sites with different magnitudes of internal fields. Because of limited statistics, however, it is difficult to decompose $P_{\mu}^{\mathrm{z}}$ observed above $T_{\mathrm{c}}$ into a sum of two exponential functions having different decay rates. Thus, previous results in Refs. [7,8] were analyzed by using a single exponential relaxation function for the electron spin contributions. We follow this approach in the analysis of the present data, which allows direct comparison of the $T_{1}$ results with the previous measurements. Level crossing resonance with nuclear quadrupole oscillation was found in $\mathrm{MnSi}$ around $B_{\mathrm{L}}=100 \mathrm{G}$ [10]. We carefully avoided this $B_{\mathrm{L}}$ in the present study.

We performed temperature scans for $B_{\mathrm{L}}=0-6000 \mathrm{G}$ and magnetic field scans at $T=30,31$, and $32 \mathrm{~K}$. Figure 1(b) shows the time spectra above $T_{\mathrm{c}}$ for $B_{\mathrm{L}}=$ $2115 \mathrm{G}$. The depolarization rate increases with decreas$\operatorname{ing} T$, reflecting the slowing down of spin fluctuations. We also observed a pronounced dependence of the time spectra on $B_{\mathrm{L}}$, as shown in Fig. 1(c). With increasing field, the signal relaxes more slowly. For the case of fast spin fluctuations with the rate $\nu \gg \gamma_{\mu} B_{\mathrm{L}} \quad\left(\gamma_{\mu}=2 \pi \times\right.$ $13.54 \mathrm{MHz} / \mathrm{kG}$ ), the relaxation rate is $1 / T_{1}=\omega^{2} / \nu$, where $\omega=\gamma_{\mu} B_{\text {int }}$ is the Larmor precession frequency of the muon spins around the instantaneous local magnetic field, $B_{\text {int }}$. To estimate $\omega$, we used $\omega=2 \pi f$, where $f=28.07(7) \mathrm{MHz}$ and $f=12.249(6) \mathrm{MHz}$ are the precession frequencies at $6 \mathrm{~K}$ [8]. The resulting spin fluctuation rate for the minimal $T_{1}=0.38 \mu \mathrm{s}$ is in the range 2.25-12.4 $\mathrm{ns}^{-1}$, which is much higher than the Larmor precession frequency $\omega_{L}=\gamma_{\mu} B_{\mathrm{L}}=0.5 \mathrm{~ns}^{-1}$ for $B_{\mathrm{L}}=$ $6 \mathrm{kG}$. Therefore, the observed field dependence of $P_{\mu}^{\mathrm{z}}(t)$ close to $T_{\mathrm{c}}$ is not due to the Zeeman level splitting of muons, but it reflects the change of the electronic spin fluctuation rate caused by $B_{\mathrm{L}}$.

In Fig. 2(a) we present the relaxation rate $1 / T_{1}$ as a function of temperature and the applied field $B_{\mathrm{L}}$. As seen in the figure, a magnetic field of $4000 \mathrm{G}$ completely suppresses the critical behavior, but for magnetic fields up to $2700 \mathrm{G}$ we still observe critical behavior near $T_{\mathrm{c}}$.

According to the SCR theory [5], the muon spin relaxation time $T_{1}$ is related to the uniform susceptibility through the relations

$$
\frac{1}{T_{1}}=\frac{\hbar \gamma_{\mu}^{2} A_{\mathrm{hf}}^{2}}{2 \pi T_{\mathrm{A}}} \frac{3 t}{2 y},
$$
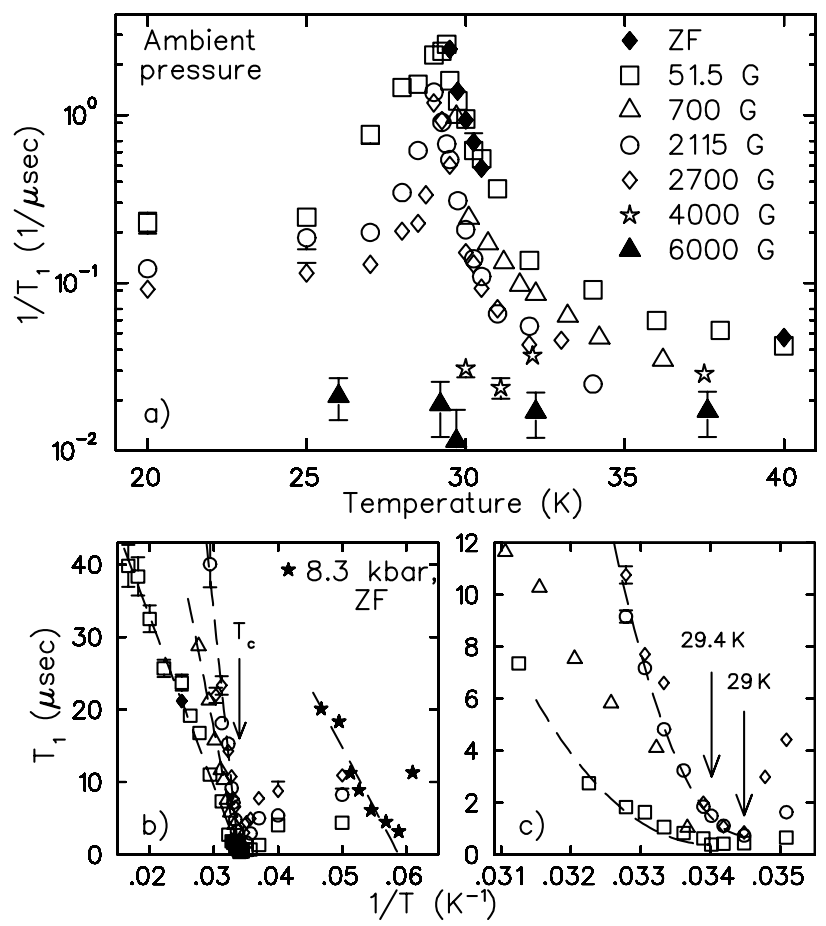

FIG. 2. (a) Temperature dependence of the relaxation rate $1 / T_{1}$ in $\mathrm{MnSi}$ at ambient pressure for $B_{\mathrm{L}}=0-6000 \mathrm{G}$. The results for $B_{\mathrm{L}}=700 \mathrm{G}$ were taken from [7]. (b) A plot of $T_{1}$ versus $1 / T$ for the results in $\mathrm{MnSi}$ at ambient pressure and under $p=8.3 \mathrm{kbar}$ (star symbol). (c) The relaxation time versus inverse temperature plot around $T_{\mathrm{c}} \approx 29.5 \mathrm{~K}$, together with the fits to $T_{1} T \propto\left(T-T_{\mathrm{c}}\right)^{2}$ shown by the broken line. 
where $y=1 /\left(2 T_{\mathrm{A}} \chi\right)$ is the reduced inverse susceptibility, $t=T / T_{0}$ is the reduced temperature, and $A_{\mathrm{hf}}$ is the muon hyperfine coupling constant. $T_{0}$ and $T_{\mathrm{A}}$ characterize the energy width of the dynamical spin fluctuation spectrum and the width of the distribution of static susceptibility in the $q$ space, respectively [11]. In the temperature region where $\chi$ obeys a Curie-Weiss law, we expect $T_{1} T$ to depend linearly on $\left(T-T_{\mathrm{c}}\right)$, which leads to a linear relationship when $T_{1}$ is plotted against the inverse temperature $1 / T$.

Figure 2(b) shows such a plot of $T_{1}$ vs $1 / T$. In the paramagnetic phase, the relaxation time depends linearly on the inverse temperature in a wide temperature region under each applied magnetic field. The slope of the line fitted to these data points increases with increasing applied field up to $B_{\mathrm{L}}=2115 \mathrm{G}$. The results reported by Hayano et al. [7] for $B_{\mathrm{L}}=700 \mathrm{G}$ are consistent with the trend found in our measurements.

Close to the critical temperature, the uniform susceptibility of itinerant systems deviates from the Curie-Weiss law, and instead follows $1 / \chi \propto\left(T-T_{\mathrm{c}}\right)^{2}$ [5], which results in $T_{1} T \propto\left(T-T_{\mathrm{c}}\right)^{2}$. There is also an intermediate region between the high temperature region and the region around $T_{\mathrm{c}}$ where $1 / \chi \propto\left(T^{4 / 3}-T_{\mathrm{c}}^{4 / 3}\right)$, which is a weakly superlinear dependence of $1 / \chi$ on temperature [5]. As $T$ approaches $T_{\mathrm{c}}$, the spin fluctuation modes with small wave vectors and low energies become predominant in the very itinerant systems, such as MnSi. This feature causes the superlinear behavior of $\chi$ and $T_{1} T$ in the critical region.

Improved experimental conditions allowed us to define the sample temperature with sufficient accuracy $( \pm 0.02 \mathrm{~K})$ around $T_{\mathrm{c}}$ to study this behavior. Figure 2(c) shows the plot of $T_{1}$ versus $1 / T$ around $T_{\mathrm{c}}$, together with a fit to the $\left(T-T_{\mathrm{c}}\right)^{2}$ dependence, with $T_{\mathrm{c}}=29.4$ and $29.0 \mathrm{~K}$ at 51.5 and $2115 \mathrm{G}$, respectively. The $T_{1}$ vs $1 / T$ plot shows nonlinear behavior also in the results obtained under 8.3 kbar, as shown in Fig. 2(b).

The magnetic field dependence of $T_{1}$ was not noticed or considered in earlier $\mu \mathrm{SR}$ studies of $\mathrm{MnSi}[7,8]$. Because the helical magnetic structure has such a long period, we first look into theoretical predictions for a weak ferromagnet. We calculate the expected values of $T_{1}\left(B_{\mathrm{L}}\right)$ in $\mathrm{MnSi}$ using the most recent approach of the SCR theory [12] with the parameters $T_{\mathrm{c}}=29.5 \mathrm{~K}$, $T_{0}=231 \mathrm{~K}, T_{\mathrm{A}}=2080 \mathrm{~K}$, and $F=3520 \mathrm{~K}$, where $F$ represents the renormalized coupling constant between the spin fluctuation modes and can be determined from the Arrott plot.

The result of these calculations, shown in Fig. 3(a), indicates that even a magnetic field as small as $700 \mathrm{G}$ would have suppressed the divergent behavior of $1 / T_{1}$ due to critical fluctuations. Comparisons of Figs. 2(a) and 3(a) clearly demonstrate that the observed divergent behavior of $1 / T_{1}$ in $700 \mathrm{G} \leq B_{\mathrm{L}} \leq 2700 \mathrm{G}$ cannot be explained by the SCR theory for a weak ferromagnet.
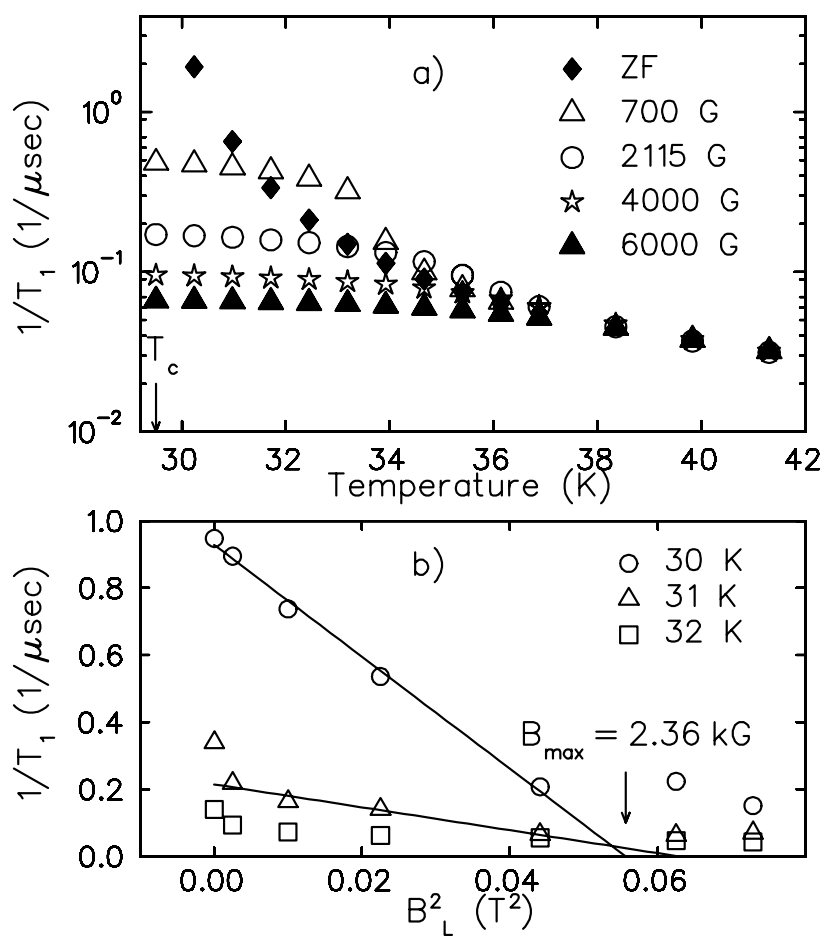

FIG. 3. (a) Temperature and magnetic field dependence of the relaxation rate calculated using the SCR model for a weak ferromagnet. (b) The measured relaxation rate at $30-32 \mathrm{~K}$ plotted versus the square of the applied magnetic field.

To explain this behavior we propose a simple model which takes into account two distinct contributions to muon spin relaxation: (a) the $q=0$ component of spin fluctuations along the direction of the external magnetic field and (b) the spin fluctuations of the remaining helical component in the plane perpendicular to the magnetic field. Accordingly, the muon spin relaxation rate in $\mathrm{MnSi}$ can be expressed as $1 / T_{1}=\left(1 / T_{1}\right)_{\text {parallel }}+\left(1 / T_{1}\right)_{\text {perp }}$. In view of $1 / T_{1} \propto \omega^{2} / \nu$ and $\omega=\gamma_{\mu} B_{\text {int }}$, the first term should be proportional to the component $S_{z}^{2}$ of $\mathrm{Mn}$ spin fluctuations parallel to the magnetic field applied along the $z$ direction and the second term proportional to $S_{\mathrm{x}, \mathrm{y}}^{2}$. Here we assume that $S_{z}^{2} \propto B_{\mathrm{L}}^{2} / B_{\max }^{2}$ and $S_{x, y}^{2} \propto$ $\left(B_{\max }^{2}-B_{\mathrm{L}}^{2}\right) / B_{\max }^{2}$, where $B_{\max }$ denotes the value of $B_{\mathrm{L}}$ required to eliminate the helical component of critical spin fluctuations.

In the ordered state at $T=4.2 \mathrm{~K}$, the external field $B_{\mathrm{L}}=6.2 \mathrm{kG}$ is sufficient to eliminate the helical component, aligning all the spins ferromagnetically, as shown in Fig. 1(a). Although it is difficult to have an accurate a priori estimate for $B_{\max }$ in the paramagnetic state, we expect that $B_{\max }$ is of the order of magnitude of $6.2 \mathrm{kG}$. According to Ref. [13], lack of inversion symmetry in an itinerant ferromagnet could create a long-period helical spin density wave. This might explain the helical spin fluctuations in MnSi persisting above $T_{\mathrm{c}}$.

Since the critical behavior due to ferromagnetic spin fluctuations is suppressed above $T=30 \mathrm{~K}$ at 


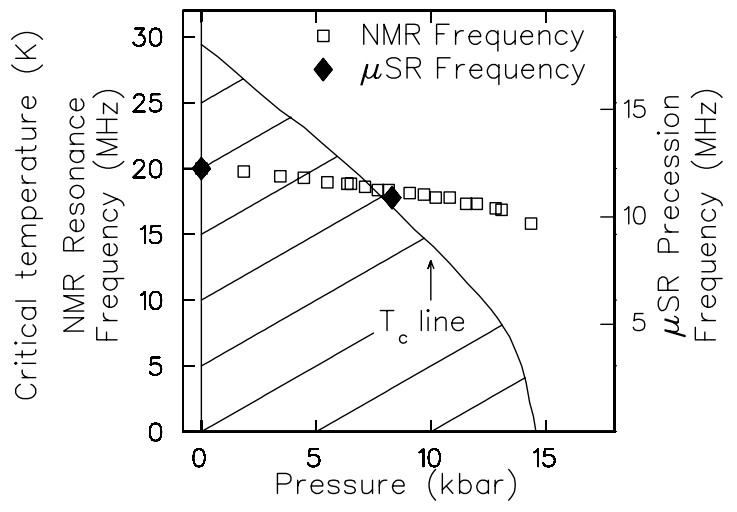

FIG. 4. Pressure dependence of the NMR frequency and $T_{\mathrm{c}}$ [14] in $\mathrm{MnSi}$. The present $\mu \mathrm{SR}$ result at $5 \mathrm{~K}$ is plotted with the rhombic symbol together with the lowest temperature precession frequency obtained by Kadono et al. [8].

$B_{\mathrm{L}} \geq 700 \mathrm{G}$, as shown in Fig. 3(b), we assume that $\left(1 / T_{1}\right)_{\text {parallel }}$ is negligibly small above $T=30 \mathrm{~K}$. Then, the observed value of $1 / T_{1}$ would solely reflect the contribution from helical spin fluctuations, leading to $1 / T_{1} \propto\left(B_{\max }^{2}-B_{\mathrm{L}}^{2}\right)$.

In Fig. 3(b) we show the observed relaxation rate $1 / T_{1}$ as a function of $B_{\mathrm{L}}^{2}$ at 30,31 , and $32 \mathrm{~K}$. The results at 31 and $32 \mathrm{~K}$ are not quite conclusive, but the magnetic field dependence of $1 / T_{1}$ at $30 \mathrm{~K}$ confirms the above prediction. From the linear fit we obtained $B_{\max }=2.36 \mathrm{kG}$. The fit for the data at $31 \mathrm{~K}$ is also consistent with this value of $B_{\max }$, except for the lowest field point. As can be seen in Fig. 1(a), the magnetic field of 2 to $3 \mathrm{kG}$ near $T_{\mathrm{c}}$ would alter the ordered state from helical to ferromagnetic. Therefore, the value of $B_{\max }$ obtained in our experiment seems reasonable. This result indicates that even in the paramagnetic state above $T_{\mathrm{c}}$, the system "knows" to which ordered structure it will transform with decreasing temperature for a given value of $B_{\mathrm{L}}$. The application of uniform field $B_{\mathrm{L}}$ suppresses the spin fluctuation of the $q=0$ component selectively, while the helical critical fluctuations survive up to $B_{\mathrm{L}}=B_{\max }$.

Another interesting issue of the SCR theory is the crossover from itinerant ferromagnet to a correlated paramagnet. Since MnSi embodies this crossover in applied pressure, extensive studies have been performed by magnetic susceptibility, resistivity [6], and NMR [14] measurements to explore regions near the quantum critical point at $p_{\mathrm{c}}$. Figure 4 shows the published results for the pressure dependence of $T_{\mathrm{c}}$ and of $M_{0}$ estimated from the ${ }^{29} \mathrm{Si}$ NMR frequency [14]. The use of single crystal specimens in $\mu \mathrm{SR}$ would assure homogeneity of the applied pressure, while powder specimens of NMR could be subject to possible inhomogeneity of pressure.

In the ordered phase, we measured the muon precession frequency under zero magnetic field. The precession disappears completely at $17 \mathrm{~K}$, where $T_{1}$ showed minimum in Fig. 2(b). Our result of $T_{c}=17 \mathrm{~K}$ for $8.3 \mathrm{kbar}$ is consistent with $T_{c}(p)$ in Fig. 4. We distinguished two precession frequencies. The results for the higher frequency have a large systematic error due to limited statistics and small precession amplitude compared to the large background signal from the pressure cell. In Fig. 4, we plot our results of the lower frequency under $8.3 \mathrm{kbar}$ at $T=5 \mathrm{~K}$, together with the value at ambient pressure. Our results confirm earlier NMR results and show that magnetic moment $M_{0}$ decreases much more slowly with increasing pressure, compared to the reduction of $T_{\mathrm{c}}$. This result might be related to the first-order nature of the phase transition at $p_{\mathrm{c}}[6,14]$.

In conclusion, we presented new sets of $\mu$ SR measurements in $\mathrm{MnSi}$ and elucidated the effect of the applied field on the critical behavior observed via the relaxation rate $1 / T_{1}$. We also found near $T_{\mathrm{c}}$ a departure of $1 / T_{1}$ from the linear behavior $T_{1} T \propto\left(T-T_{\mathrm{c}}\right)$ and confirmed the very small pressure dependence of the magnetic moment in the ordered phase.

We thank G. Shirane for providing a large single crystal specimen of MnSi and T. Moriya and Y. Takahashi for helpful discussions on the SCR theory. The work was supported by NSF-DMR-0102752 and CHE0117752 (at Columbia), DMR-9731361 and DMR0102293 (at Riverside), and U.S. DOE/BES (at Los Alamos).

[1] Y. Ishikawa, K. Tajima, D. Bloch, and M. Roth, Solid State Commun. 19, 525 (1976).

[2] Y. Ishikawa, G. Shirane, J. A. Tarvin, and M. Kohgi, Phys. Rev. B 16, 4956 (1977).

[3] E. Fawcett, J. P. Maita, and J. H. Wernick, Int. J. Magn. 1, 29 (1970).

[4] H. Yasuoka, V. Jaccarino, R. C. Sherwood, and J. H. Wernick, J. Phys. Soc. Jpn. 44, 842 (1978).

[5] T. Moriya, Spin Fluctuations in Itinerant Electron Magnetism, Springer Series in Solid State Sciences Vol. 56 (Springer, Heidelberg, 1985).

[6] C. Pfleiderer, G. J. McMullan, S. R. Julian, and G. G. Lonzarich, Phys. Rev. B 55, 8330 (1997).

[7] R. Hayano et al., J. Phys. Soc. Jpn. 49, 1773 (1980).

[8] R. Kadono, T. Matsuzaki, T. Yamazaki, S. R. Kreitzman, and J. H. Brewer, Phys. Rev. B 42, 6515 (1990).

[9] M. Takigawa et al., J. Phys. Soc. Jpn. 49, 1760 (1980).

[10] R. Kadono et al., Phys. Rev. B 48, 16803 (1993).

[11] A. Ishigaki and T. Moriya, J. Phys. Soc. Jpn. 65, 3402 (1996).

[12] A. Ishigaki and T. Moriya, J. Phys. Soc. Jpn. 67, 3924 (1998).

[13] O. Nakanishi, A. Yanase, A. Hasegawa, and M. Kataoka, Solid State Commun. 35, 995 (1980).

[14] C. Thessieu, Y. Kitaoka, and K. Asayama, Physica (Amsterdam) 259-261B, 847 (1999). 